\title{
Conformations de polymères fondus dans des pores très petits
}

\author{
F. Brochard et P. G. de Gennes \\ Laboratoire de Physique de la Matière Condensée, \\ Collège de France, 11, place Marcelin-Berthelot, \\ 75231 Paris Cedex 05, France \\ (Reçu le 8 mai 1979, accepté le 25 juin 1979)
}

\begin{abstract}
Résumé. - On discute ici les conformations de chaînes flexibles pour une phase fondue, injectée dans un pore cylindrique de diamètre $D$ inférieur à la taille naturelle $\left(R_{0}=N^{1 / 2} a\right)$ des chaînes $(N=$ nombre de monomères par molécule). On trouve que pour $D \gg N^{1 / 4} a$ la chaîne a une dimension $R_{\|}$le long de l'axe du tube qui est non perturbée $\left(R_{\|}=R_{0}\right)$. Par contre pour $D \ll N^{1 / 4} a$, la dimension $R_{\|}$croît $\left(R_{\|} \cong N a^{3} / D^{2}\right)$. Ces résultats sont généralisés ensuite au cas où la chaîne étudiée $(N)$ est incorporée dans une matrice de chaînes de longueur différente $(P \neq N)$.

Abstract. - We discuss theoretically the conformations of individual chains in a melt, when the melt is confined in a cylindrical pore with a diameter $D$ smaller than the natural size $\left(R_{0}=N^{1 / 2} a\right)$ of the chains ( $N$ being the number of monomers per chain). For $D \gg N^{1 / 4} a$, we find a longitudinal dimension $R_{\|}$of the chain which is unperturbed $\left(R_{\|}=R_{0}\right)$. For $D \ll N^{1 / 4} a$, the size $R_{\|}$increases $\left(R_{\|} \cong N a^{3} / D^{2}\right)$. We also generalize these results to the case of one chain (with $N$ monomers) immersed in a matrix of chains with a different length ( $P$ monomers/chain).
\end{abstract}

Les propriétés de chaînes confinées dans des tubes ont été examinées (au niveau des lois d'échelle) pour différents régimes de concentration, dans les réfs. [1] (conformations) et [2] (dynamique). Toutefois, l'analyse de la réf. [1] pour les conformations d'une chaîne dans le cas concentré s'avère incorrecte : le but de la présente note est de corriger cette erreur, puis d'en tirer certaines conséquences sur la dynamique. Physiquement, l'hypothèse cruciale revient toujours à supposer des parois passives (pas d'adsorption des chaînes).

1. Conformations pour un système monodisperse. Pour un diamètre de tube $D$ très grand, on retourne à un cas tridimensionnel bien connu [4] : en phase fondue, des chaînes toutes de même longueur sont idéales et ont une taille $R_{0}=N^{1 / 2} a$. Partant de cette situation, faisons décroître $D$ jusqu'à une valeur inférieure à $R_{0}$. Chaque chaîne est maintenant confinée dans deux directions. Nous voulons trouver son extension $\left(R_{\|}\right)$dans la $3^{\mathrm{e}}$ direction, parallèle à l'axe du tube. Supposons d'abord que la chaîne considérée reste décrite par une marche aléatoire simple dans cette direction $\left(R_{\|}=R_{0}\right)$. Pour examiner la validité de cette hypothèse, nous étudierons deux paramètres principaux :
- le taux de remplissage $\varphi_{\text {int }}$ : la chaîne est inscrite dans un volume $D^{2} R_{\|} \rightarrow D^{2} R_{0}$ et son volume propre est d'ordre $\mathrm{Na}^{3}$. Donc

$$
\varphi_{\mathrm{int}} \cong N a^{3} /\left(D^{2} R_{0}\right) \cong N^{1 / 2}(a / D)^{2}
$$

- le paramètre de perturbation $\zeta$ : défini comme dans Yamakawa [3] qui donne la force des effets de répulsion stérique à l'intérieur d'une chaîne $(\zeta<1$ correspond à une chaîne idéale, $\zeta>1$ à une chaîne gonflée). Dimensionnellement on peut écrire

$$
\zeta \cong \varphi_{\mathrm{int}} N v a^{-3}
$$

où $v$ est le volume exclu par monomère. Pour une chaîne unique, en très bon solvant, on aurait $v=a^{3}$ (volume du monomère). Mais ici, en phase fondue, l'interaction $v$ est réduite par un effet d'écran, dû aux autres chaînes présentes [4-6], et prend la forme

$$
v=a^{3} N^{-1} \text {. }
$$

Les éqs. (3) et (2) montrent que dans le cas présent

$$
\zeta \cong \varphi_{\text {int }} \text {. }
$$

Revenant alors à l'éq. (1) on aboutit aux conclusions suivantes :

a) pour $D \gg N^{1 / 4} a, \varphi_{\text {int }}$ et $\zeta$ sont petits : il est alors correct de supposer la chaîne idéale $\left(R_{\|}=R_{0}\right)$; 
b) pour $D \ll N^{1 / 4} a$ on ne peut plus avoir $R_{\|}=R_{0}$ car on aboutirait à $\varphi_{\text {int }}>1$, ce qui est inacceptable. Il est naturel de supposer que, lorsque $D$ décroît (à $N$ fixé), la valeur réelle de $\varphi_{\text {int }}$ croît de façon monotone : donc $\varphi_{\text {int }}$ se sature à sa valeur maximum $\varphi_{\text {int }} \rightarrow 1$ et ceci impose

$$
R_{\|} \cong N a^{3} D^{-2} .
$$

Dans le régime $(b)$ les chaînes sont spatialement ségrégées ; chaque chaîne occupe une certaine longueur de tube, et les chaînes se suivent consécutivement comme des voitures dans un tunnel. Une chaîne et sa voisine ne se recouvrent que dans une région commune étroite. Notons que (5) est en désaccord avec la réf. [1] qui prédisait $R_{\|} \sim N a^{2} / D$ dans la limite concentrée. On avait introduit dans la réf. [1] des sous-unités ou blobs de taille $D$ et supposé implicitement que ces sous-unités avaient une interaction fortement répulsive, donc qu'elles se disposaient côte à côte sans recouvrement. En réalité, les blobs ainsi définis peuvent s'interpénétrer : l'analogue du paramètre $\zeta$ défini pour un blob est inférieur à 1 , même dans le régime $(b)$.

2. Généralisation. - Considérons maintenant une chaîne $(N)$ immergée dans une matrice fondue de chaînes chimiquement identiques, mais de longueur différente (degré de polymérisation $P$ ). Ici l'analyse des réfs. [4-6] montre que l'interaction écrantée prend la forme :

$$
v=a^{3} P^{-1}
$$

et l'éq. (4) est donc remplacée par

$$
\zeta \cong \varphi_{\text {int }} N P^{-1}
$$

Dans ce cas $\zeta$ et $\varphi_{\text {int }}$ sont donc des paramètres indépendants. On trouve alors cinq régimes (selon les valeurs de $N$ et $P$ ) représentés sur la figure 1 .

(I) chaîne $(N)$ idéale dans un tube grand

(II) chaîne $(N)$ confinée mais encore idéale $\left(R_{\|}=R_{0}\right)$

(III) chaîne $(N)$ ségrégée (éq. (5))

(IV) chaîne $(N)$ gonflée par l'interaction $\left(3^{\prime}\right)$, mais de taille $\widetilde{R}_{\mathrm{F}}$ inférieure au diamètre du tube

(V) chaîne $(N)$ gonflée et confinée.

Pour le régime (IV) on peut utiliser l'analyse de Flory [4] et on obtient (dans la limite du gonflement fort, qui correspond à $N \gg P^{2}$ )

$$
\tilde{R_{\mathrm{F}}} \cong a N^{3 / 5} P^{-1 / 5} \text {. }
$$

Pour le régime $(\mathrm{V})$ on obtient la taille $R_{\|}$à partir d'une énergie libre $F_{N}$ inspirée de Flory, mais prenant $D^{2} R_{\|}$comme volume global de la pelote

$$
F_{N} / k T \cong \frac{R_{\|}^{2}}{R_{0}^{2}}+\frac{N^{2} a^{3}}{P D^{2} R_{\|}}
$$

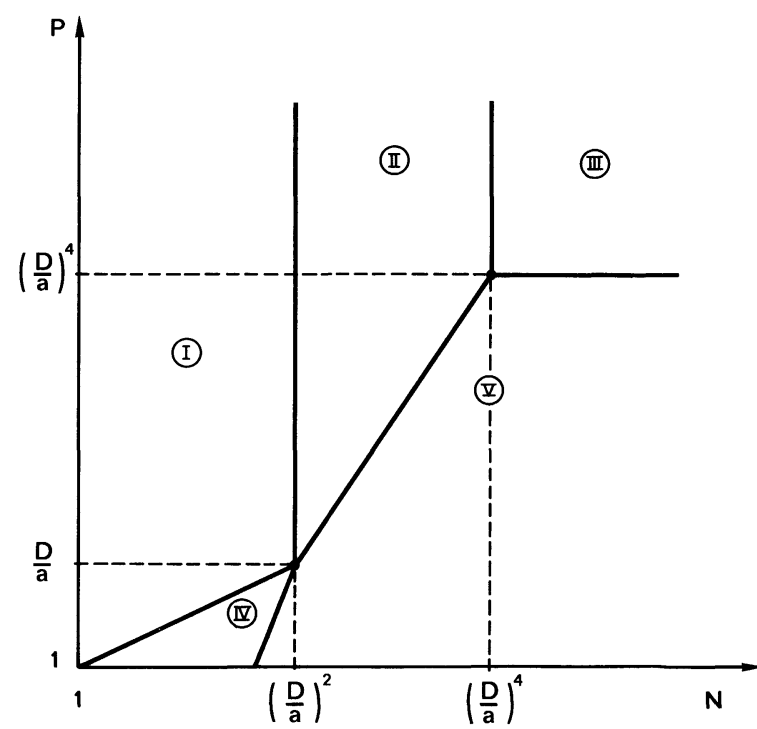

Fig. 1. - Cinq régimes pour les conformations d'une chaîne (de $N$ monomères) immergée dans un polymère fondu ( $P$ monomères/chaîne). Le système est confiné dans un tube de diamètre $D$ fixé. La longueur $(a)$ est la taille du monomère. (Les échelles en $P$ et $N$ sont logarithmiques.) Noter que les frontières entre régimes sont en fait diffuses.

[Five regimes for the conformations of one chain ( $N$ monomers) in a melt ( $P$ monomers/chain). The melt is confined inside a tube of fixed diameter $D$. The scales (for $N$ and $P$ ) are logarithmic. The meaning of the different regimes is explained after eq. $\left(4^{\prime}\right)$. Note that the boundaries between different regimes are not sharp, but diffuse.]

Le premier terme est l'énergie élastique d'allongement (dans la limite d'allongement fort). Le deuxième terme représente l'interaction $\left(3^{\prime}\right)$ calculée avec la densité interne $\varphi_{\text {int }} . T$ est la temipérature absolue, $k$ la constante de Boltzmann. Tous les coefficients numériques sont ignorés. Après minimisation de (7) par rapport à $R_{\|}$on trouve

$$
R_{\|}=N a\left[a^{2} / P D^{2}\right]^{1 / 3} .
$$

Le domaine de validité de (8) est indiqué sur la figure 1. Notons que le régime (V) n'est pas observable dans le cas monodisperse $(N=P)$.

3. Conclusions. - a) En bon solvant, une chaîne unique s'allonge dès que le diamètre $D$ du tube est inférieur à sa taille naturelle [1]. Au contraire, dans une matrice de chaînes fondues identiques $(N=P)$ la chaîne ne doit pas s'allonger dès que $D$ devient inférieur à $R_{0}$, mais seulement à une valeur plus faible de $D\left(\sim N^{1 / 4} a\right)$. Toute la discussion de la section IV dans la réf. [1] doit être revue dans ce sens.

b) Quand $R_{\|}>R_{0}$, l'allongement des chaînes doit avoir des conséquences dynamiques intéressantes. Par exemple, dans le cas $N=P$, si $D>N^{1 / 4} a$, une description de reptation [7] doit s'appliquer sans modification grave. Par contre, pour $D<N^{1 / 4} a$, 
la situation paraît plus subtile : à cause de la disposition en voitures dans un tunnel des différentes chaînes, il existe une barrière qui s'oppose au dépassement d'une voiture par une autre. Toutefois, après examen, comme $\zeta \sim 1$ dans ce régime, la barrière serait d'ordre $k T$, donc franchissable. Si on accepte cette proposition, le modèle de reptation conduit à un coefficient de self diffusion

$$
D_{\mathrm{s}} \cong R_{\|}^{2} / \tau_{\text {rep }}
$$

où $\tau_{\text {rep }}$ est le temps de reptation, et doit être peu sensible au confinement :

$$
\tau_{\text {rep }} \cong \tau_{1} N^{3} N_{\mathrm{e}}^{-1}
$$

Dans l'éq. (10) $\tau_{1}$ est un temps microscopique, et
$N_{\mathrm{e}}$ le nombre de monomères entre points d'enchevêtrement [8]. A partir de (9), (10) et (5) on aboutit à

$$
D_{\mathrm{s}} \cong a^{2} \tau_{1}^{-1}\left(\frac{a}{D}\right)^{4} N_{\mathrm{e}} N^{-1}
$$

Donc pour $D<N^{1 / 4} a$, on retrouverait un coefficient de diffusion proportionnel à $N^{-1}$. (Bien entendu, ce résultat suppose que les chaînes ne sont pas attachées aux parois.)

c) L'ensemble de ces prédictions reste très conjectural, mais devrait pouvoir être comparé soit à des calculs numériques, soit à des expériences sur des chaînes incorporées dans des nucléopores, ou dans certaines phases hexagonales.

\section{Bibliographie}

[1] Daoud, M., De Gennes, P. G., J. Physique 38 (1977) 85.

[2] Brochard, F., De Gennes, P. G., J. Chem. Phys. 67 (1977) 52.

Brochard, F., J. Physique 38 (1977) 1285.

[3] Yamakawa, H., Modern Theory of Polymer Solutions (Harper and Row, NY) 1972 ;

Domb, C., Barrett, A., Polymer 17 (1976) 179.
[4] Flory, P., J. Chem. Phys. 17 (1949) 303.

[5] Edwards, S. F., Proc. Phys. Soc. 88 (1966) 265.

[6] De Gennes, P. G., J. Polym. Sci. Symposia 61 (1977) 313.

[7] De Gennes, P. G., J. Chem. Phys. 55 (1971) 572.

[8] Noter que pour $N \sim N_{\mathrm{e}}$ l'éq. (10) redonne un temps de Rouse $\tau_{\text {rep }} \rightarrow \tau_{1} N^{2}$. 